\title{
Study of Chromium Migration in Nickel-based Alloy using Polarized Neutron Reflectometry and Rutherford Backscattering Spectrometry
}

\author{
Mathieu Doucet, ${ }^{*}, 1$ James F. Browning, ${ }^{1}$ Barney L. Doyle, ${ }^{2}$ Timothy R. Charlton, ${ }^{1}$ Haile Ambaye,${ }^{1}$ \\ Joohyun Seo, ${ }^{1}$ Alessandro R. Mazza, ${ }^{3}$ John F. Wenzel, ${ }^{1}$ George R. Burns,${ }^{2}$ Ryan R. Wixom, ${ }^{4}$ and \\ Gabriel M. Veith ${ }^{5}$ \\ ${ }^{1}$ Neutron Scattering Division, Oak Ridge National Laboratory, Oak Ridge, TN 37831, United States \\ ${ }^{2}$ Radiation-Solid Interactions Department, Sandia National Laboratories, Albuquerque, NM 87185, United States \\ ${ }^{3}$ Materials Science and Technology Division, Oak Ridge National Laboratory, Oak Ridge, TN 37831, United States \\ ${ }^{4}$ Center for Integrated Nanotechnologies, Sandia National Laboratories, Albuquerque, NM 87123, United States \\ ${ }^{5}$ Chemical Sciences Division, Oak Ridge National Laboratory, Oak Ridge, TN 37831, United States \\ *Corresponding author: doucetm@ornl.gov
}

\section{NOTICE OF COPYRIGHT}

This manuscript has been authored by UT-Battelle, LLC under Contract DE-AC05-00OR22725 with the U.S. Department of Energy (DOE). The U.S. government retains and the publisher, by accepting the article for publication, acknowledges that the US government retains a nonexclusive, paid-up, irrevocable, worldwide license to publish or reproduce the published form of this manuscript, or allow others to do so, for U.S. government purposes. DOE will provide public access to these results of federally sponsored research in accordance with the DOE Public Access Plan (http://energy.gov/downloads/doe-public-accessplan). 

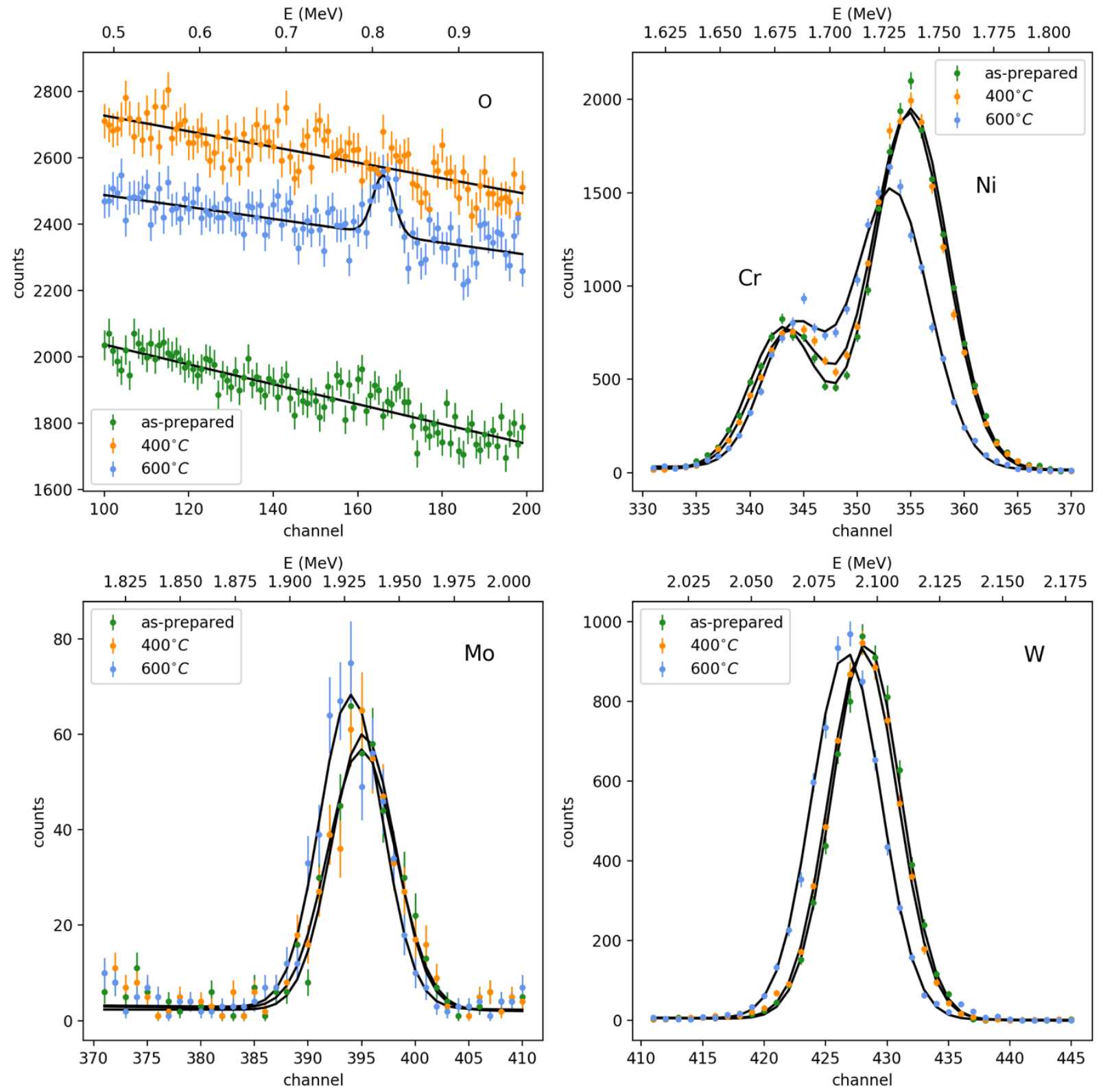

Figure S1 RBS energy spectra for oxygen, chromium, nickel, molybdenum, and tungsten. 

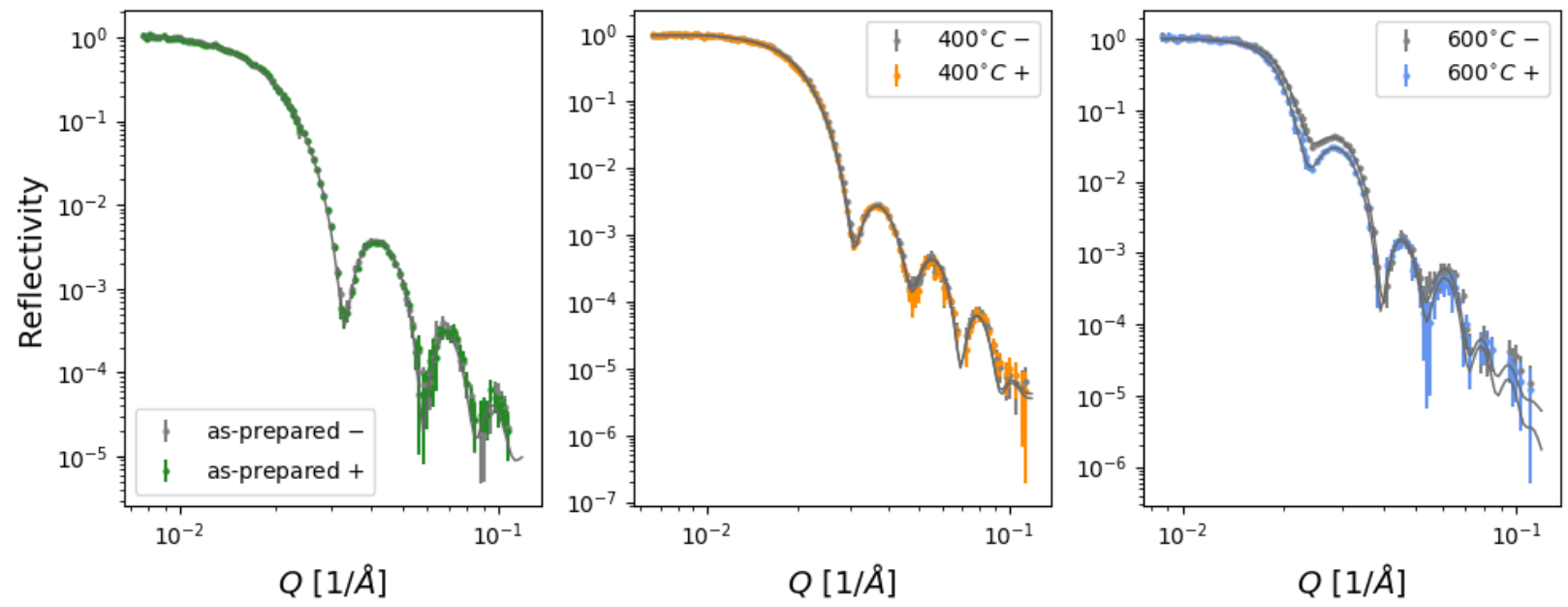

Figure S2 Measured reflectivity in each spin state for the as-prepared, $400^{\circ} \mathrm{C}$, and $600^{\circ} \mathrm{C}$ samples.
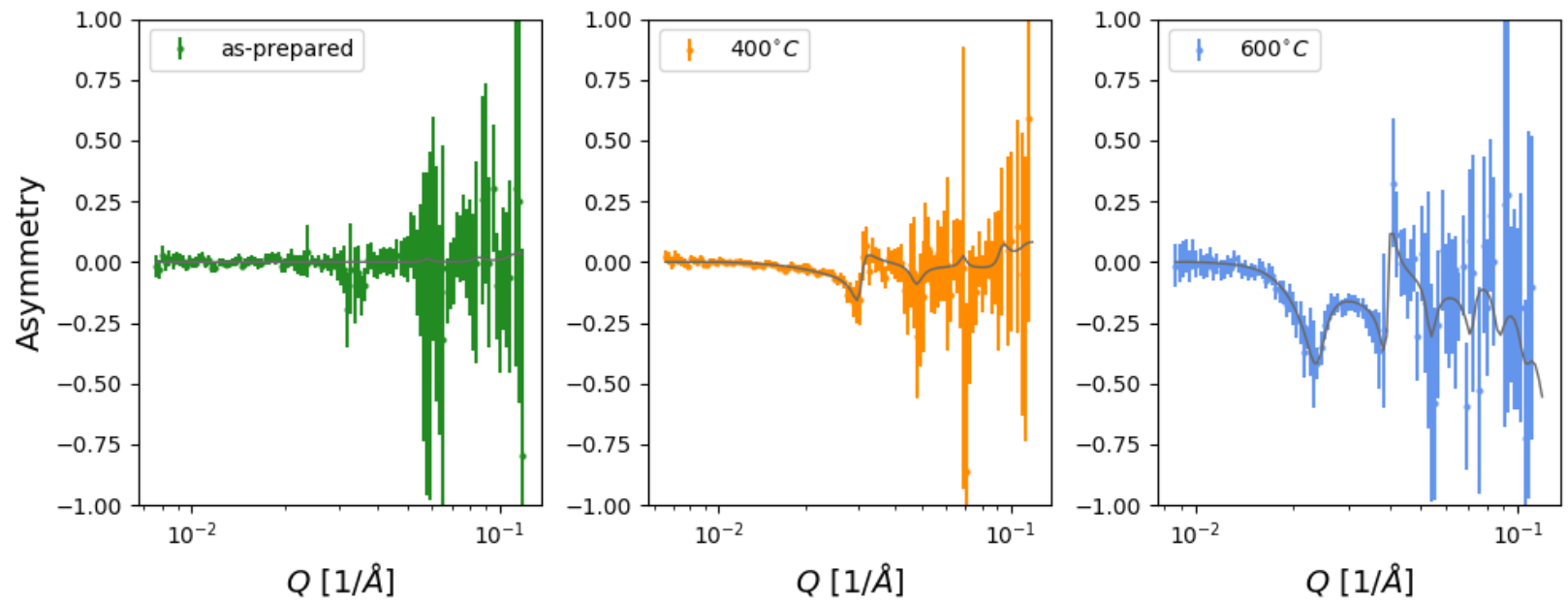

Figure S3 Spin state asymmetry measurements for the as-prepared, $400^{\circ} \mathrm{C}$, and $600^{\circ} \mathrm{C}$ samples. 
Table S1 Model parameter including a magnetic component to the oxide layer.

\begin{tabular}{lll}
\hline Sample & $\mathbf{4 0 0}^{\circ} \mathbf{C}$ & $\mathbf{6 0 0}^{\circ} \mathbf{C}$ \\
\hline Oxide thickness $[\AA \mathbf{\AA}]$ & $78.8 \pm 2.9$ & $201.9 \pm 2.9$ \\
Oxide nuclear SLD $\left[\mathbf{1 0}^{-6} / \AA^{2}\right]$ & $4.4 \pm 0.4$ & $4.76 \pm 0.09$ \\
Oxide magnetic SLD $\left[\mathbf{1 0}^{-6} / \AA^{2}\right]$ & $-0.007 \pm 0.025$ & $0.039 \pm 0.052$ \\
Oxide roughness $[\AA]$ & $19.4 \pm 3.6$ & $21.8 \pm 2.8$ \\
Bulk thickness $[\AA]$ & $197.0 \pm 4.8$ & $162.5 \pm 2.7$ \\
Bulk nuclear SLD $\left[\mathbf{1 0}^{-6} / \AA^{2}\right]$ & $6.59 \pm 0.03$ & $7.10 \pm 0.07$ \\
Bulk magnetic SLD $\left[\mathbf{1 0}^{-6} / \AA^{2}\right]$ & $0.076 \pm 0.007$ & $0.41 \pm 0.03$ \\
Bulk roughness $[\AA]$ & $17.4 \pm 7.1$ & $23.6 \pm 4.6$ \\
Substrate roughness $[\AA]$ & $18.5 \pm 1.0$ & $12.9 \pm 2.2$ \\
\hline \hline$\chi^{\mathbf{2} / \text { dof }}$ & 1.150 & 0.724 \\
\hline
\end{tabular}

Table S2 Surface composition of target material and test thin film sample compared to vendor specifications. Compositions are given in weight percent [wt\%] and atomic percent [at $\%$ ]. The composition of the thin film measured with EDS is also given in atomic percent for comparison with the Ion Beam Analysis.

\begin{tabular}{llllll}
\hline Element & $\begin{array}{l}\text { Target } \\
{[\mathbf{w t} \%]}\end{array}$ & $\begin{array}{l}\text { Thin Film } \\
{[\mathbf{w t} \%]}\end{array}$ & $\begin{array}{l}\text { Thin Film } \\
{[\mathbf{a t \%} \text { ] }}\end{array}$ & $\begin{array}{l}\text { RBS as-prepared } \\
{[\mathbf{a t \%} \%}\end{array}$ & $\begin{array}{l}\text { Specification } \\
{[\mathbf{w t} \%]}\end{array}$ \\
\hline Nickel & $68.5 \pm 4.3$ & 63.5 & 65.8 & $63.6 \pm 6.4$ & $>57$ \\
Chromium & $14.8 \pm 9.2$ & 21.9 & 25.6 & $32.6 \pm 3.3$ & 22 \\
Tungsten & $10.9 \pm 3.2$ & 8.6 & 2.8 & $3.1 \pm 0.6$ & 14 \\
Molybdenum & $1.3 \pm 0.7$ & 1.2 & 0.8 & $0.7 \pm 0.5$ & 2.0 \\
Iron & $2.0 \pm 1.0$ & 1.5 & 1.6 & - & 3 \\
Aluminum & $1.2 \pm 4.0$ & 1 & 2.3 & - & 0.3 \\
Silicon & $0.4 \pm 3.0$ & - & - & - & - \\
Manganese & $0.5 \pm 1.3$ & 1 & 1.1 & - & 0.5 \\
\hline
\end{tabular}

